\title{
A Multi-dimensional Evaluation on Capacity Matching Between High-speed Railway and Subway
}

\author{
Peiran Han $^{a}$ and Yu Zhao ${ }^{b}$ \\ School of Traffic and Transportation, Beijing Jiao tong University, Beijing, 100044, China. \\ a17120807@bjtu.edu.cn, byuzhao@bjtu.edu.cn
}

Keyword: comprehensive transportation hub, high speed railway, subway, capacity matching, transit.

\begin{abstract}
This paper mainly analyzed transit capacity matching between high speed railway and subway in comprehensive transportation hub. Combined with example and used TOPSIS method to evaluate the Beijing south railway station capacity matching condition, proposed reasonable suggestions to improve current situation.
\end{abstract}

\section{Introduction}

High speed railway and subway play crucial role in comprehensive transportation hub, however, connection between two traffic mode always has a unconsistent performance in hubs of China, which caused problems like insufficient or surplus capacity. Compared scale of them, high speed railway retains predominance all the time. with a certain amount of railway capacity, subway can be adjusted dynamically to optimize the effect of matching. In this way, passenger flow in hub is more efficient and transfer capacity is improved. Guo [1] evaluated from area attribute, network environment, passenger flow, the type of transfer connection and the equipment capacity. Zhang [2] studied with transfer mode, character of layout and information system. Liang [3] set up a matching model of evacuation capability with optimized plan. This paper mainly analyzed capacity matching from each part of hub.

\section{Capacity Matching and Factor}

Capacity matching is the matching degree between two traffic modes in a broader view, which is the most direct index to evaluate transfer capacity and efficiency. In previous studies, the ratio of two modes capacity in a period of time was divided into several ranks, which is called capacity matching degree.

Capacity matching between railway and subway in comprehensive hub is mainly reflected in whether subway transit can evacuate the passenger flow rapidly at rail arrival peak. In addition to considering connection of transportation capacity parameters in the hub, it is also necessary to consider the matching relationship between the capacity of transfer channel and station equipment. To sum up, the main influencing factors are mainly:

(1) Interval between two traffic modes

The time interval of different modes of transportation directly affects transfer efficiency and hub transport organization. A bad match will result in a waste of the operator's capacity, and passengers will be stranded on platform. The time interval matches well, subway can quickly evacuate passenger flow during rush hour.

(2) The optimization of streamline layout

The capacity of equipment in the transfer line, the crowding degree of directly influences the degree of capacity matching. Design of streamline should avoid the conflict between different types and different directions.

(3) The influence of transfer mode.

Beijing south railway station adopts the hall transfer mode, the passenger flow organization efficiency is relatively high, the transfer speed is faster. If the passengers stay too long in hall, the 
number of assembly in the transfer hall will increase, which affect transfer efficiency and organization.

\section{Multi-Dimensional Evaluation of Capacity Matching}

Due to the complexity of integrated hub, it is necessary to evaluate matching from different

Dimensions. Summarized objective operating indicators from serval aspects. This paper mainly uses TOPSIS method.

\subsection{Data Processing}

TOPSIS evaluation is a multi-dimensional evaluation method. There are mainly the following types of data processing methods for different dimensions and trends:

(1) Trend treatment: the index of the optimal direction is the benchmark, and the index of different trends can be taken inverse.

(2) Normalization processing: for the dimension of the unified index, the number of $0-1$ is processed, the formula is as follows:

$$
\begin{gathered}
D^{+}=\sqrt{\sum_{1}^{n}\left(a_{i j}^{+}-a_{i j}\right)^{2}} \\
D^{-}=\sqrt{\sum_{1}^{n}\left(a_{i j}^{-}-a_{i j}\right)^{2}}
\end{gathered}
$$

According to the above method, the optimal and worst vectors can be determined, and the distance and proximity between each evaluation object and the ideal solution $C_{\mathrm{i}}$ can be calculated, whose range is between 0 and 1 . The closer result get to 1 , the vector is better. According to each $\bar{C}_{i}$, the merits and demerits of the evaluation plan can be sorted out.

\subsection{Index Selection}

This paper mainly selects indicators from the following dimensions: the ratio of passenger flow and subway transport capacity of subway station in the peak hour is used to reflect the degree of matching; The impact of the peak passenger flow on the equipment with the equipment fitness; Use ratio of passenger who transfer inside to reflect the macroscopic transfer capability.

(1) Capacity matching degree: capacity matching $0.86-0.90$ is optimal, but south station data at around 0.5 , more than 0.90 it is not realistic, so regulate this index for the bigger the optimal;

(2) Fitness equipment: This index is the bigger the better while it is below 1, Beijing south railway station equipment fitness [6] is about 0.4-0.5;

(3) Inner-station transfer proportion: It reflects whether the subway platform remaining ability is enough to evacuate passengers, which is one of the most important indicators objectively reflect the capacity matching degree.

\section{Capacity Matching Evaluation-A Case Study in Beijing South Railway Station}

\subsection{Transfer Analysis}

In the transfer hall of Beijing south railway station, the passengers Beijing-Tianjin inter-City train are mainly from the arrivals gate 7 and 8 , and the rest from 3, 4 and 6 . It can be seen that the conflict of the transfer hall is mainly in the high-speed rail entrance and the arrival port. When the two transfer routes are arriving at the same time, it is easy to cause congestion at the entrance of the subway. The main stream line conflict is near the entrance of the subway, and the flow that reaches at the port of 7 and 8 to the north bus hub has the maximum interference to the streamline. And when the subway passenger, there will be interference with the exit passenger flow. According to the above analysis, combined with the actual situation, it is concluded that there are some problems in the operation of Beijing south railway station:

(1) Transfer facility Settings.

The over-concentration of transfer facilities in transfer line can lead to a large concentration of passenger flow. The number is unreasonable, leading to the bottleneck of transportation. The 
connection between different parts of the transfer facilities is not close enough, which will make capacity matching and passenger flow characteristics are not obvious.

(2) Streamline conflict

There are various flow lines in integrated transportation hub, and if the conflicts of different kinds of streamlines cannot be effectively unsolved, it will seriously affect organization. The flow line inside metro pay zone is the same path, which belongs to the conflict of flow line in different direction.

(3) Hub features

During the working day, the passenger flow is more stable, the morning and evening peak passenger flow is fixed, and the high-speed railway reaches the peak at 13:30-15:00 in the afternoon. The weekend's peak season is mainly on Friday and Sunday evening.

\subsection{Capacity Matching Analysis}

\subsubsection{Subway Transmission Capacity}

The transportation capacity is 210 for each carriage and 6 for marshalling. The departure interval of line 4 is $2 \mathrm{~min}$. Line 14 is $4 \mathrm{~min} 30 \mathrm{~s}$. The maximum capacity of line 4 is $120 \%$, and the transmission coefficient is 0.6 at rush hour, and line 14 is the terminal, taking 1 . The maximum number of people in the urban rail transit in Beijing south railway station is:

$$
\begin{aligned}
& N_{4 \max \text { board }}=2 \times 1260 \times 120 \% \times 0.6 \times\left(\frac{60}{2}\right)=54432 \\
& C_{\text {platform }}=1188 \times 0.6 \times 3.78=2694
\end{aligned}
$$

\subsubsection{Bottleneck Identification}

According to the standard of subway design, the capacity of each transfer facility in Beijing south railway station is investigated and the capacity of the inbound direction is calculated. The following table shows the layout of various equipment in Beijing south railway station.

Table 1. Beijing South Subway Station Facility

\begin{tabular}{ccccc}
\hline Location & stairwidth & Direction & Escalator width \\
\hline Hall -B2 & $3 \mathrm{~m}$ & Both way & Two escalators, intermediate stairs. & \\
\cline { 1 - 3 } B2-B3 & $3 \mathrm{~m}$ & Both way & Four platforms & $1 \mathrm{~m}$ \\
\cline { 1 - 3 } Hall-B3 & $4 \mathrm{~m}$ & $\begin{array}{c}\text { Both way } \\
\text { (separated) } \\
\text { Both way }\end{array}$ & $\begin{array}{c}\text { Two escalators, intermediate stairs. } \\
\text { One escalator and stairs }\end{array}$ & \\
\hline
\end{tabular}

(1) Gate

There are two subway entrances in the transfer hall and 12 gates can serve.

$$
C_{\text {gate }}=2100 \times 12=25200(\mathrm{p} / \mathrm{h})
$$

(2) Stairs and escalators

There are 4 escalators in line 4 of Beijing south subway station, two escalators go up and two go down, and two stairs are $3 \mathrm{~m}$ wide. Line 14 has four escalators, which has the same layout with line 4 , and two both-way stairs with four meters wide. So, the capacity of the stairs and escalators can be calculated:

(3) Platform

$$
C_{\text {stairs }}^{4-14}=3200 \times 3=9600(\mathrm{p} / \mathrm{h})
$$

The effective length of the subway platform is $108 \mathrm{~m}$, and the effective width is $11 \mathrm{~m}$, thus area is $1188 \mathrm{~m} 2$ [4]. The maximum passenger density is $3.78 \mathrm{p} / \mathrm{m} 2$ [5], with a capacity of 0.6 , so the capacity of the platform is:

$$
C_{\text {platform }}=1188 \times 0.6 \times 3.78=2694
$$


(4) Dynamic bottleneck

The identification of dynamic bottleneck is mainly used to analyze the fitness of equipment. The fitness of equipment reflects the degree of coordination between passenger flow and fixed equipment through capacity. The calculation formula is as follows:

$$
B=\frac{Q}{C}
$$

In the formula: $\mathrm{Q}$ is the change of passenger flow at rush hour;

$\mathrm{C}$ is the ability to use fixed equipment.

The static capacity of the transfer facility in the inbound passenger flow direction is in turn: $N_{4 \max \text { board }}>C_{\text {楼梯 }}^{4}>C_{\text {埇机 }}$, To see the ability of line 4 stops the minimum value is 25200 (person/hour), maximum hours into the station passenger flow at 9749 Beijing south station, so stop brake machine capacity is enough, streamline can meet the demand of the service.

\subsection{Case Study-TOPSIS}

Based on the survey data from March 12 to 18, 2017, this paper established the TOPSIS evaluation matrix, as shown in the following table:

Table 2. Line 4 Capacity Matching Evaluation with TOPSIS

\begin{tabular}{ccccc}
\hline Line 4 & Matching degree & Facility fitness & Staie fitness & Inner proportion \\
\hline Mar 12th & 0.56 & 0.60 & 0.27 & 0.35 \\
Mar 13th & 0.39 & 0.40 & 0.19 & 0.78 \\
Mar 14th & 0.39 & 0.40 & 0.19 & 0.82 \\
Mar 15th & 0.42 & 0.50 & 0.21 & 0.78 \\
Mar 16th & 0.44 & 0.50 & 0.21 & 0.73 \\
Mar 17th & 0.57 & 0.60 & 0.28 & 0.57 \\
Mar 18th & 0.41 & 0.40 & 0.20 & 0.51 \\
\hline
\end{tabular}

After normalization, select the optimal vector $\mathrm{A}+$ and the worst vector A-:

$$
\begin{aligned}
& A^{+}=\{0.4686,0.4602,0.4686,0.4615\} \\
& A^{-}=\{0.3192,0.3068,0.3192,0.1968\}
\end{aligned}
$$

Table 3. Evaluation Result

\begin{tabular}{ccccc}
\hline Line 4 & $\mathrm{D}+$ & $\mathrm{D}-$ & $\mathrm{C}_{\mathrm{i}}$ & Ranking \\
\hline Mar 12th & 0.2628 & 0.2948 & 0.5287 & 4 \\
Mar 13th & 0.3206 & 0.2446 & 0.4328 & 6 \\
Mar 14th & 0.3180 & 0.2653 & 0.4548 & 5 \\
Mar 15th & 0.2143 & 0.2740 & 0.5611 & 3 \\
Mar 16th & 0.1925 & 0.2621 & 0.5766 & 2 \\
Mar 17th & 0.1398 & 0.3435 & 0.7108 & 1 \\
Mar 18th & 0.3250 & 0.1097 & 0.2524 & 7 \\
\hline
\end{tabular}

From the above table, the best evaluation results were on Friday, 17 March, with minimum distance between the indexes and the best indicators. The matching degree of the capacity and the fitness of the gate are optimal. The transfer ratio in subway station is 0.57 . In this ratio, it is a good match. The passenger flow on this day is the peak of the sample, the subway can effectively evacuate the passenger flow. However, combined with the capacity matching degree table, 0.57 is only a general performance, and capacity matching condition of line 4 is still not ideal and needs to be improved.

There are some ways to improve the capacity matching method mainly to adjust the train marshalling and departure interval. However, in combination with the actual situation, passengers can't get to the subway platform in time due to detention and congestion during the transfer. Line 4 
departure intervals is $1 \mathrm{~min}$, line 14 is $4 \mathrm{~min} 30 \mathrm{~s}$, changing departure interval measures is not feasible, should find out congestion areas and bottleneck region, improve overall transfer ability.

\subsection{Improve Capacity Matching Measures}

(1) Strengthen guidance and isolation measures in the two-way transfer equipment to prevent the occurrence of congestion and dangerous conditions.

(2) Increase the number of ticket machines in the subway during peak. Avoid a large backlog of passengers at the entrance.

(3) Two operation and management departments should set up a reasonable departure plan to realize seamless connection.

\section{Summary}

As the main driving force of the integrated transportation hub, the matching of high-speed rail and urban rail transit restricts the development of the hub. Based on the literature at home and abroad research on the change to the cohesion, analyzed three influence factors influencing the capacity matching, used TOPSIS method to Beijing south railway station capacity matching do comprehensive evaluation, in view of the objective operation indicators, got the optimal index vector. The optimal index is analyzed to make suggestions for operation management.

\section{References}

[1]. Guo, J. Evaluation and application research on integrated railway hub transfer [D]. Beijing: Beijing Jiao tong University, 2010.

[2]. Zhang, H. Research on transfer between railway and subway [D]. Chengdu: Southwest Jiao tong University, 2007.

[3]. Liang, Y and Zhang, X. Study on the coordination and allocation scheme of the transport capacity of integrated railway passenger terminal [J]. Railway computer application, 2009, 18(9):11-15.

[4]. Wang, G. Study on simulation model of urban rail transit platform passenger aggregation [D]. Beijing: Beijing Jiao tong University, 2012.

[5]. Fu, T. Identification of bottleneck of urban rail transit station dispersion capacity [D]. Beijing: Beijing Jiao tong University,2014.

[6]. Ministry of construction, People's Republic of China. Subway design and planning GB 504157-2003[M]. Beijing: China planning press, 2003. 